\title{
Heat Transfer Coefficient of an Under-Expanding Cold Spray Air Jet on a Flat Substrate
}

\author{
Amirhossein Mahdavi, André McDonald \\ Department of Mechanical Engineering, University of Alberta \\ 10-203 Donadeo Innovation Center for Engineering, Edmonton, Alberta T6G 1H9, Canada \\ amahdavi@ualberta.ca; andre.mcdonald@ualberta.ca
}

\begin{abstract}
A two-dimensional heat conduction model was developed to determine the transient temperature distribution within a flat substrate that was exposed to the impingement of a cold spray hot air jet during the deposition process. The credibility of employing average heat transfer coefficient in mathematical modelling and prediction of the surface temperature profile of a substrate was studied. Moreover, the condition under which the effect of the presence of the in-flight particles on the heat transfer coefficient of the underexpanding air jet can be neglected was investigated. In this regard, a cold spray unit was used to generate a supersonic air jet. A twodimensional heat conduction model was developed and solved by using Green's functions to determine the temperature distribution within the substrate. By applying a surface integral to the analytically-estimated spatially-varying heat transfer coefficient of an underexpanding cold spray air jet, the average heat transfer coefficient was determined. Both the average and spatially-varying heat transfer coefficients were used separately in the model to predict the transient surface temperature profile of the substrate. It was shown that when the Stokes number of the particles is sufficiently small, the effect of the presence of the particles on the heat transfer coefficient of the impinging dilute air-particle jet can be neglected. It was further found that the surface temperature that was predicted by using the average heat transfer coefficient, unlike the spatially-varying heat transfer coefficient, produced large errors, especially at higher distances from the stagnation point of the air jet on the substrate surface.
\end{abstract}

Keywords: Air-Particle Jet Impingement, Average Heat Transfer Coefficient, Cold Spraying, Temperature Distribution.

\section{Introduction}

Cold spraying is a coating fabrication process in which a supersonic air jet is generated to accelerate and direct micron-sized powder particles toward the substrate in order to form a dense and adherent coating [1]. The quality and the deposition efficiency of the final coatings are significantly affected by substrate surface heating during the thermal or cold spraying process [2, 3]. For instance, Watanabe, et al. [3] investigated the effect of surface temperature on the adhesion strength of cold-sprayed coatings. It was found that increasing the substrate temperature resulted in higher adhesion strength of copper $(\mathrm{Cu})$ coatings on A5083 aluminum, iron $(\mathrm{Fe})$, and $\mathrm{Cu}$ substrates. Therefore, investigation of the gassubstrate heat transfer becomes important.

Several studies have been conducted to investigate the heat exchange between the impinging air jet that was generated by a cold spray nozzle and the substrate [4 - 6]. Mahdavi and McDonald developed a semi-empirical analytical model to predict the spatially-varying heat transfer coefficient between an under-expanding air jet and a flat substrate during cold spraying $[4,5]$. The aforementioned model was capable of predicting the transient surface temperature profile of the substrate. On the other hand, a few numerical and analytical studies have modelled the gas-substrate heat exchange by applying an analytically-estimated external average heat flux, or by using average heat transfer coefficient to evaluate the heat flux [7]. In this regard, a challenging issue may arise as to whether or not employing average heat transfer coefficient in the mathematical modelling considerably influences on the determination of the surface temperature profile of the target substrate. Moreover, in the aforementioned studies on the prediction of the heat transfer coefficient, the effect of the presence of the in-flight particles on the heat transfer coefficient was neglected. However, in some cases, the existence of the particles may affect the flow pattern and the heat transfer coefficient of the air jet [8].

The main objectives of the current study were to (i) evaluate the conditions in which the influence of the particles on heat transfer coefficient could be neglected, (ii) employ the analytical heat conduction model to determine the surface temperature profile for average and spatially-varying heat transfer coefficient, and (iii) compare the analytically-estimated 
and experimentally-measured surface temperature to evaluate the suitability of using the average heat transfer coefficient to determine the transient surface temperature of the substrate that was exposed to an impinging cold spray air jet.

\section{Experimental Procedure}

A low-pressure cold spray unit (SST Series P, Centerline, Ltd., Windsor, ON, Canada) and a programmed robot (Motoman-HP20, Yaskawa Electric Corp., Waukegan, IL, USA) were employed to generate and direct a supersonic air jet. The distance between the nozzle exit and the substrate was $15 \mathrm{~mm}$. The substrate was a disk-shape aluminum 6061-T6. The information on the substrate and the cold spray system parameters that were used in this study are summarized in Table 1. The back surface of the substrate was insulated by attaching a thermal insulation material, vitreous aluminosilicate fiber (ISAFORM $2300^{\circ}$ L.O., Insulation Specialties of America, Inc., Wanatah, IN, USA) to prevent it from exchanging heat with the ambient air. The transient temperatures of the surface of the substrate were measured by using a calibrated infrared camera (FLIR A65, FLIR Systems, Inc., Nashua, NH, USA). According to the manufacturer, the bias uncertainty of the camera measurement was $\pm 5^{\circ} \mathrm{C}$ or $5 \%$ of the reading. In order to obtain accurate measurements during the experiments, the front surface of the smooth substrate was painted black so as to approximate the conditions of a black body. Subsequently, the emissivity of the substrate material was set to 0.96 on the infrared camera.

Table 1: Information on substrate and cold spray parameters.

\begin{tabular}{|c|c|}
\hline Air temperature & $300^{\circ} \mathrm{C}$ \\
\hline Air pressure & $660 \mathrm{kPa}$ \\
\hline Nozzle exit diameter $\left(D_{\mathrm{n}}\right)$ & $6.3 \mathrm{~mm}$ \\
\hline Substrate diameter $(2 b)$ & $200 \mathrm{~mm}$ \\
\hline Substrate thickness $(\delta)$ & $1.5 \mathrm{~mm}$ \\
\hline Substrate thermal conductivity $\left(k_{\mathrm{s}}\right)$ & $167 \mathrm{~W} / \mathrm{m}-\mathrm{K}$ \\
\hline
\end{tabular}

\section{Analytical Method}

Due to the high velocity of the under-expanding hot air jet over the substrate, a significant amount of thermal energy was presumed to be transferred from the impinging air jet to the flat substrate. A two dimensional heat conduction model was developed to evaluate the temperature distribution within the substrate that was exposed to the impingement of a cold spray air jet. The impinging air jet was considered to produce an external heat flux, creating a boundary condition of third kind. The schematic of the geometry of the mathematical model is shown in Fig. 1.

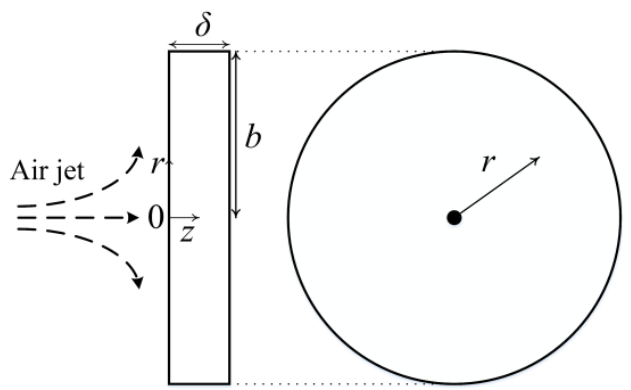

Fig. 1: Schematic of the mathematical model [5].

The governing equation for the transient three-dimensional heat conduction model is

$$
\frac{1}{r} \frac{\partial}{\partial r}\left(r \frac{\partial T(r, z, t)}{\partial r}\right)+\frac{\partial^{2} T(r, z, t)}{\partial z^{2}}=\frac{1}{\alpha_{\mathrm{s}}} \frac{\partial T(r, z, t)}{\partial t}
$$

where $\alpha_{\mathrm{s}}$ is the thermal diffusivity of the substrate material. The boundary conditions and initial condition are 


$$
\begin{gathered}
T(r=0, z, t)=\text { finite, } \\
\frac{\partial T(r=b, z, t)}{\partial r}=0, \\
-k_{\mathrm{s}}\left[\frac{\partial T(r, z=0, t)}{\partial z}\right]=h(r)\left[T_{\mathrm{AW}}(r)-T(r, z=0, t)\right], \\
\frac{\partial T(r, z=\delta, t)}{\partial z}=0, \\
T(r, z, t=0)=T_{0} .
\end{gathered}
$$

In Eq. (4) $k_{\mathrm{s}}$ is the thermal conductivity of the substrate material. The external heat flux on the front surface of the substrate is given by Eq. (4), where $h$ is the spatially-varying forced convective heat transfer coefficient between the front surface and the under-expanding air jet, and $T_{\mathrm{AW}}$ represents the adiabatic wall temperature of the impinging air jet over the substrate [4]. The adiabatic wall temperature $\left(T_{\mathrm{AW}}\right)$ takes into account the effect of viscous dissipation during the impingement and spreading of a compressible high-velocity air jet upon the substrate. In Eq. (6) $T_{0}$ represents the initial temperature of the substrate. Green's functions were employed in solution of the heat conduction governing equation and the final expression for the temperature distribution within the substrate was found to be

$$
\begin{aligned}
T(r, z, t) & =\sum_{i=1}^{\infty} \sum_{j=1}^{\infty}\left[T_{0} \frac{\exp \left[-\alpha_{\mathrm{s}}\left(\varsigma_{\mathrm{i}}^{2}+\beta_{\mathrm{j}}^{2}\right) t\right]}{N\left(\varsigma_{\mathrm{i}}\right) N\left(\beta_{\mathrm{j}}\right)} J_{0}\left(\varsigma_{\mathrm{i}} r\right)\left[\tan \left(\beta_{\mathrm{j}} \delta\right) \sin \left(\beta_{\mathrm{j}} z\right)+\cos \left(\beta_{\mathrm{j}} z\right)\right]\right. \\
& \times \int_{r^{\prime}=0}^{b} \int_{z^{\prime}=0}^{\delta} J_{0}\left(\varsigma_{\mathrm{i}} r^{\prime}\right)\left[\tan \left(\beta_{\mathrm{j}} \delta\right) \sin \left(\beta_{\mathrm{j}} z^{\prime}\right)+\cos \left(\beta_{\mathrm{j}} z^{\prime}\right)\right] r^{\prime} d z^{\prime} d r^{\prime}+\left(\frac{\alpha_{\mathrm{s}}}{k_{\mathrm{s}}}\right)\left[\frac{h(r)}{N\left(\varsigma_{\mathrm{i}}\right) N\left(\beta_{\mathrm{j}}\right)}\right] J_{0}\left(\varsigma_{\mathrm{i}} r\right) \\
& \left.\times \frac{\left[\tan \left(\beta_{\mathrm{j}} \delta\right) \sin \left(\beta_{\mathrm{j}} z\right)+\cos \left(\beta_{\mathrm{j}} z\right)\right]}{\alpha_{\mathrm{s}}\left(\varsigma_{\mathrm{i}}^{2}+\beta_{\mathrm{j}}^{2}\right)}\left[1-\exp \left(-\alpha_{\mathrm{s}}\left(\varsigma_{\mathrm{i}}^{2}+\beta_{\mathrm{j}}^{2}\right) t\right)\right] \int_{r^{\prime}=0}^{b} J_{0}\left(\varsigma_{\mathrm{i}} r^{\prime}\right) T_{\mathrm{AW}}\left(r^{\prime}\right) r^{\prime} d r^{\prime}\right],
\end{aligned}
$$

where $J$ represents the Bessel function of the first kind. In Eq. (7), $\zeta_{\mathrm{i}}$ and $N\left(\zeta_{\mathrm{i}}\right)$ are the eigenvalues and Norm of the differential equation in the radial coordinate $(0 \leq r \leq b)$, respectively, and can be calculated as

$$
\begin{gathered}
\varsigma_{\mathrm{i}} J_{1}\left(\varsigma_{\mathrm{i}} b\right)=0, \\
N\left(\varsigma_{\mathrm{i}}\right)=\int_{r=0}^{b} J_{0}^{2}\left(\varsigma_{\mathrm{i}} r\right) r d r .
\end{gathered}
$$

In Eq. (7), $\beta \mathrm{j}$ and $N(\beta \mathrm{j})$ are the eigenvalues and Norm of the differential equation in the axial coordinate $(0 \leq z \leq \delta)$, respectively, and can be calculated from

$$
\beta_{\mathrm{j}} \tan \left(\beta_{\mathrm{j}} \delta\right)-\frac{h(r)}{k_{\mathrm{s}}}=0
$$




$$
N\left(\beta_{\mathrm{j}}\right)=\int_{z=0}^{\delta}\left[\tan \left(\beta_{\mathrm{j}} \delta\right) \sin \left(\beta_{\mathrm{j}} z\right)+\cos \left(\beta_{\mathrm{j}} z\right)\right]^{2} d z
$$

Equation (7) represents the analytical solution to determine the temperature distribution within the substrate exposed to an impinging hot air jet of a cold spray unit. A zero-dimensional code in MATLAB (MathWorks, Inc., Natick, MA, USA) was developed to solve for temperature values from the explicit expression for $T(r, z, t)$.

\section{Results and Discussion}

Estimates of the heat transfer coefficient of the under-expanding air jet were required in order to determine the transient temperature distribution within the substrate. In this regard, Mahdavi and McDonald [4] developed a semiempirical analytical model to predict the spatially-varying convective heat transfer coefficient of a cold spray impinging air jet at various working temperatures and pressures of the system. Figure 2 shows the spatially-varying Nusselt number of an under-expanded air jet generated by a cold spray system at a pressure of $660 \mathrm{kPa}$ (96 psig) and temperature of $300^{\circ} \mathrm{C}$ [4]. The non-dimensional Nusselt number, $\mathrm{Nu}$, is defined as

$$
\mathrm{Nu}=\frac{h D_{\mathrm{n}}}{k_{\mathrm{g}}}
$$

where $k_{\mathrm{g}}$ is thermal conductivity of the air (working gas). The distance from the stagnation point of the impinging jet was non-dimensionalized as

$$
\eta=\frac{r}{D_{\mathrm{n}}}
$$

where $r$ is the radial distance from the stagnation point of the impinging air jet. The stagnation point of the impinging air jet is at $r=0$.

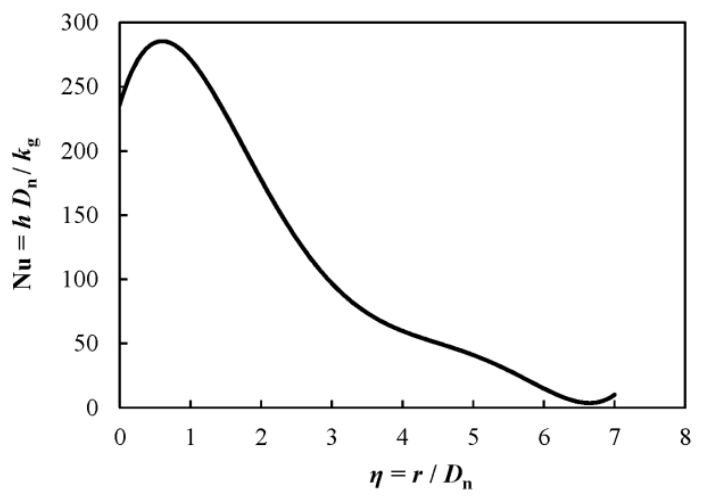

Fig. 2: Non-dimensional Nusselt number of the impinging air jet produced by a cold spray system as a function of non-dimensional radial distance from the stagnation point [4].

In the aforementioned semi-empirical analytical model that was used to determine the heat transfer coefficient of the impinging air jet [4], the effect of the presence of the particles in the air jet was neglected. However, the presence of the metal or alloy powder particles in the supersonic air jet is essential for fabrication of the coatings. Therefore, that the issue is whether or not the presence of the particles noticeably affects the convective heat transfer coefficient of the impinging air jet.

In numerical modelling of the cold spray process, it is a common assumption to neglect particle/particle collision in order to reduce the complexity and cost of the modelling, when the air-particle mixture is sufficiently dilute. Under 
experimental and practical conditions in actual cold spraying, the aforementioned assumption is valid when the volume fraction of the discrete phase (particles) in the air-particle mixture is sufficiently low [9]. Moreover, the particle-air interaction can be characterized by introducing the non-dimensional Stokes number (Stk). The Stokes number is the ratio of the momentum response time of the particle $\left(\tau_{\mathrm{p}}\right)$ to the characteristic time of the flow field $\left(D_{\mathrm{n}} / u_{\mathrm{g}}\right)$. The Stokes number is expressed as [9]

$$
\mathrm{Stk}=\tau_{\mathrm{p}} \frac{u_{\mathrm{g}}}{D_{\mathrm{n}}}
$$

where $u_{\mathrm{g}}$ represents the average velocity of the air jet. In Eq. (14), the momentum response time of the particle $\left(\tau_{\mathrm{p}}\right)$ is defined as the time that a particle requires to respond to any changes in velocity and it is expressed as [9]

$$
\tau_{\mathrm{p}}=\frac{\rho_{\mathrm{p}} d_{\mathrm{p}}^{2}}{18 \mu_{\mathrm{g}}}
$$

where $\rho_{\mathrm{p}}$ and $d_{\mathrm{p}}$ are the density and diameter of the particle, respectively, and $\mu_{\mathrm{g}}$ is the air (working gas) viscosity. For small Stokes number $(\mathrm{Stk}<1)$, the response time of the particles is less than the characteristic time of the flow field; therefore, the particles have sufficient time to respond to variations in the flow field. As a result, the velocity of the particles will be almost the same as that of the gas flow velocity. On the contrary, for large Stokes number (Stk > 1), the particle velocity will be marginally affected by the gas flow velocity [9]. Thus, it is expected that for a dilute gas-particle mixture jet with small Stokes number, the particles will follow the air jet flow pattern during the impingement and underexpansion of the air jet over the substrate [8]. In other words, the velocity and flow pattern of the air jet will likely be minimally affected by the particles. Consequently, the heat transfer coefficient that was estimated for an impinging air jet without consideration of the presence of the particles can be applied to the case of an actual cold spray air-particle mixture jet, since the heat transfer coefficient is primarily dependent on the velocity and flow pattern of the working fluid [10]. In the current study, it was assumed that the sizes of the particles were in a range such that their Stokes numbers were on the order of unity or less. Thus, since the Stokes numbers were on the order of 1 or less, the analytically-estimated heat transfer coefficient values that were found by Mahdavi and McDonald [4] were used in the current study to investigate the effect of employing average heat transfer coefficient in mathematical modelling on the substrate surface temperature profile. In this regard, the spatially-varying heat transfer coefficient was integrated over a region of $7 D_{\mathrm{n}}$ on the substrate surface, which likely covered the region of the front surface of the substrate that was most affected by the impinging air jet, according to the experimental observations. The average heat transfer coefficient $(\bar{h})$ was evaluated as

$$
\bar{h}=\frac{1}{A_{\mathrm{s}}} \int_{r=0}^{7 D_{\mathrm{n}} / 2} h(r)(2 \pi r d r),
$$

where

$$
A_{\mathrm{s}}=\frac{49 \pi D_{\mathrm{n}}{ }^{2}}{4} .
$$

The average heat transfer coefficient obtained from Eq. (16) was substituted in Eqs. (7) and (10) in order to determine the transient surface temperature profile of a substrate that was exposed to a stationary impinging air jet. The surface temperature profiles were predicted at various times in the form of non-dimensional Fourier numbers $\left(\mathrm{Fo}=\alpha_{\mathrm{s}} t / \delta^{2}\right)$ of 27, 81, and 137. Figure 3 shows comparisons of the experimentally-measured and predicted non-dimensional surface temperature profiles as a function of the distance from the stagnation point at different Fourier numbers. Both average and spatially-varying heat transfer coefficient were separately used in the mathematical model to predict the surface temperature profile. It was found that using the average heat transfer coefficient resulted in large deviations of the 
predicted substrate surface temperature from those obtained from the experiments, while the analytically-estimated surface temperature that was obtained by using the spatially varying heat transfer coefficient was in good agreement with the experimental data. Due to the spatial variation of the velocity of the under-expanding air flow over the substrate, the heat transfer coefficient was strongly dependent on the distance from the stagnation point of the jet [4]. According to Newton's law of cooling, the estimated temperature is highly dependent on the variation of the heat transfer coefficient [11]. Therefore, use of the average heat transfer coefficient would neglect the effects of spatial variation of this parameter, which in turn would produce errors in predicting the surface temperature profile of the substrate.

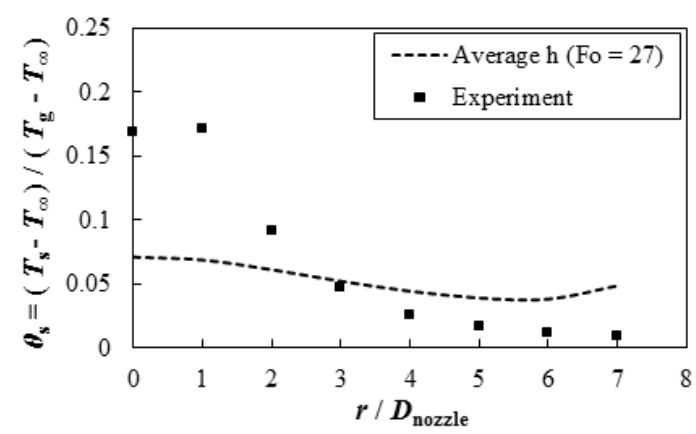

(a)

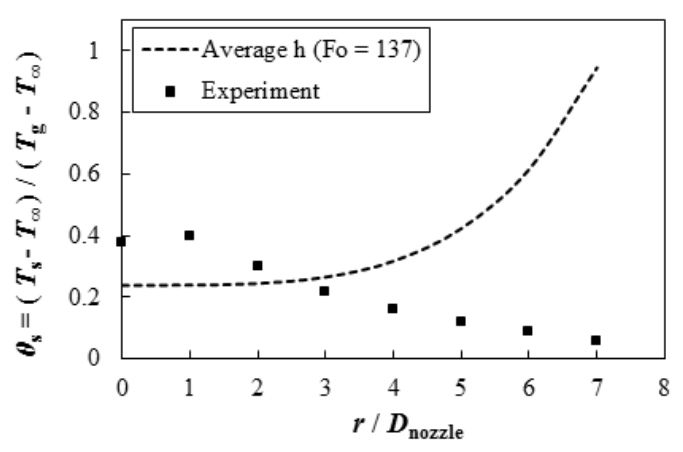

(c)

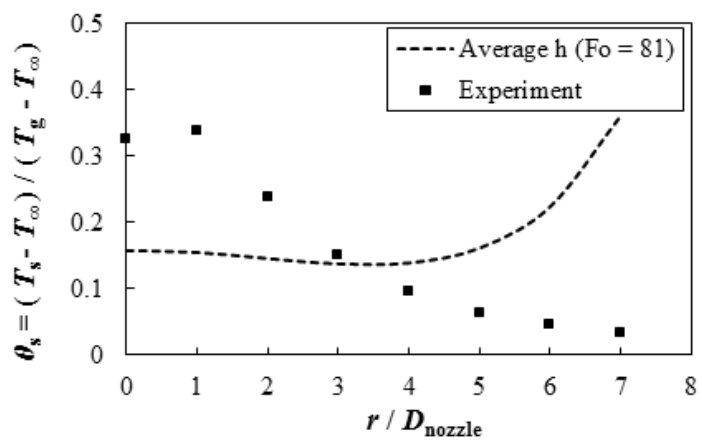

(b)

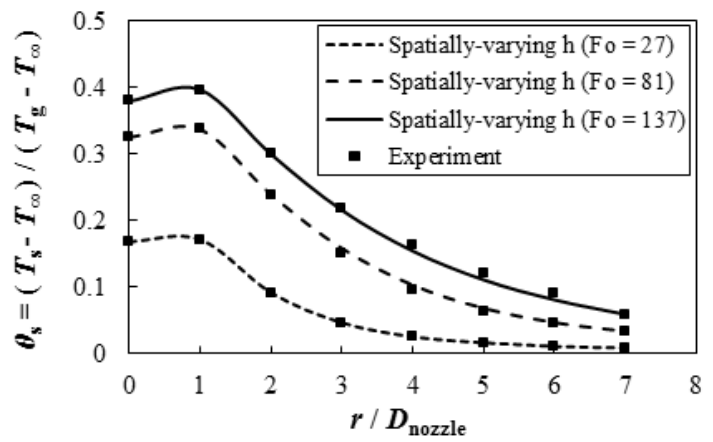

(d)

Fig. 3: Comparison of the experimental and analytical non-dimensional temperature profile at different Fourier numbers for (a) - (c) average and (d) spatially-varying heat transfer coefficient [4].

\section{Conclusion}

A two-dimensional heat conduction model was developed to assess the suitability of using average heat transfer coefficient in predicting the temperature distribution of a substrate surface during cold spraying. It was shown that under specific conditions, when the Stokes number of the particles is sufficiently small, the effect of the presence of the particles on the heat transfer coefficient of the impinging dilute air-particle jet can be neglected. It was further found that employing average heat transfer coefficient generated significant deviations between the predicted and experimental surface temperatures. It was concluded that the spatially-varying heat transfer coefficient should be used in the heat conduction model to predict the surface temperatrure of the substrate during the impingement of an actual cold spray air-particle jet with small Stokes number.

\section{Acknowledgements}

Funding for this project was provided by the Natural Science and Engineering Research Council of Canada on a Discovery Grant. A detailed, extended version of the current study has been submitted to Surface and Coatings Technology. 


\section{References}

[1] P. Fauchais, J. Heberlein and M. Boulos, Thermal Spray Fundamentals from Powder to Part. New York: Springer, 2014.

[2] M. Fukumoto, H. Wada, K. Tanabe, M. Yamada, E. Yamaguchi, A. Niwa, M. Sugimoto and M. Izawa, "Effect of substrate temperature on deposition behavior of coper particles on substrate surface in the cold spray process," $J$. Therm. Spray Technol., vol. 16, pp. 643-650, 2007.

[3] Y. Watanabe, C. Yoshida, K. Atsumi, M. Yamada, and M. Fukumoto, "Influence of substrate temperature on adhesion strength of cold-sprayed coatings," J. Therm. Spray Technol., vol. 24, no. 1-2, pp. 86-91, 2015.

[4] A. Mahdavi, and A. McDonald, "Effect of Substrate and Process Parameters on the Gas-Substrate Convective Heat Transfer Coefficient during Cold Spraying," J. Therm. Spray Technol., vol. 23, no. 3, pp. 433-445, 2018.

[5] A. Mahdavi, A. McDonald, "Analytical evaluation of temperature distribution within the substrate under an impinging cold spray hot air jet," in Proceedings of the International Thermal Spray Conference, Shanghai, People's Republic of China, pp. 1-5, 2016.

[6] A. Ryabinin, E. Irissou, A. McDonald, and J.-G. Legoux, "Simulation of gas-substrate heat exchange during coldgas dynamic spraying," Int. J. Therm. Sci., vol. 56, pp. 12-18, 2012.

[7] Y. Chen, X. Liang, Y. Liu, J. Bai and B. Xu, "Finite element modelling of coating formation and transient heat transfer in the electric arc spray process," Int. J. Heat Mass Tran., vol. 53, pp. 2012-2021, 2010.

[8] B. Samareh and A. Dolatabadi, "A three-dimensional analysis of the cold spray process: the effects of substrate location and shape," J. Therm. Spray Technol., vol. 16, pp. 634-642, 2007.

[9] C. T. Crowe, Mutiphase Flow Handbook. New York: Taylor \& Francis Group, 2006.

[10] A. Shapiro, The Dynamics and Thermodynamics of Compressible Fluid Flow, Vol. II. New York: The Ronald Press Company, 1954.

[11] L. Jiji, Heat Conduction. Second ed., New York: Begell House, 2003. 